Article

\title{
Eccentricity Based Topological Indices of an Oxide Network
}

\author{
Muhammad Imran 1,2,*, Muhammad Kamran Siddiqui 1,3 (10), Amna A. E. Abunamous ${ }^{1}$, \\ Dana Adi ${ }^{1}$, Saida Hafsa Rafique ${ }^{1}$ and Abdul Qudair Baig ${ }^{4}$ \\ 1 Department of Mathematical Sciences, United Arab Emirates University, Al Ain 15551, UAE; \\ kamransiddiqui75@gmail.com (M.K.S.); 201250600@uaeu.ac.ae (A.A.E.A.); \\ 201450261@uaeu.ac.ae (D.A.); 201350314@uaeu.ac.ae (S.H.R.) \\ 2 Department of Mathematics, School of Natural Sciences (SNS), \\ National University of Sciences and Technology (NUST), Sector H-12, Islamabad 44000, Pakistan \\ 3 Department of Mathematics, COMSATS University Islamabad, Sahiwal Campus, Sahiwal 57000, Pakistan \\ 4 Department of Mathematics, The University of Lahore, Pakpattan Campus, Pakpattan 57400, Pakistan; \\ aqbaig1@gmail.com \\ * Correspondence: imrandhab@gmail.com
}

Received: 5 June 2018; Accepted: 11 July 2018; Published: 18 July 2018

\begin{abstract}
Graph theory has much great advances in the field of mathematical chemistry. Chemical graph theory has become very popular among researchers because of its wide applications in mathematical chemistry. The molecular topological descriptors are the numerical invariants of a molecular graph and are very useful for predicting their bioactivity. A great variety of such indices are studied and used in theoretical chemistry, pharmaceutical researchers, in drugs and in different other fields. In this article, we study the chemical graph of an oxide network and compute the total eccentricity, average eccentricity, eccentricity based Zagreb indices, atom-bond connectivity $(A B C)$ index and geometric arithmetic index of an oxide network. Furthermore, we give analytically closed formulas of these indices which are helpful in studying the underlying topologies.
\end{abstract}

Keywords: molecular graph; total eccentricity; average eccentricity; eccentricity based Zagreb indices; atom bond connectivity index; geometric arithmetic index and oxide network

MSC: 05C12, 05C90

\section{Introduction}

Graph theory is a branch of mathematics that has a lot of applications in computer science, electrical systems (network), interconnected systems (network), biological networks, and in chemistry. Chemical graph theory is the rapidly developing zone among chemists and mathematicians. Chemical graph theory helps us to predict the certain physico-chemical properties of chemical compounds by just considering their pictorial representations [1,2].

Cheminformatics is a comparatively new subject, which is a combination of chemistry, mathematics and information science. There is a considerable usage of graph theory in theoretical and computational chemistry. Chemical graph theory is the topology branch of mathematical chemistry which implements graph theory to mathematically model chemical occurrences. There has been a lot of research in this area in the last few decades. A few references are given that demonstrate the significance of graph theory in Mathematical Chemistry [3,4].

Let $G=(V, E)$ be a graph, where $V$ is a non-empty set of vertices and $E$ is a set of edges. Chemical graph theory applies graph theory to the mathematical modeling of molecular phenomena, which is 
helpful for the study of molecular structures. The manipulation and examination of chemical structural information is made conceivable by using molecular descriptors. A great variety of topological indices are studied and used in theoretical chemistry by pharmaceutical researchers. In chemical graph theory, there are many topological indices for a connected graph, which are helpful in the study of chemical molecules. This theory has had a great effect in the development of chemical science.

If $p, q \in V(G)$, then the distance $d(p, q)$ between $p$ and $q$ is defined as the length of any shortest path in $G$ connecting $p$ and $q$. Eccentricity is the distance of vertex $u$ from the farthest vertex in $G$. In mathematical form,

$$
\varepsilon(u)=\max \{d(u, v) \mid \forall u \in V(G)\} .
$$

The total eccentricity index is introduced by Farooq et al. [5], which is defined as,

$$
\zeta(G)=\sum_{v \in V(G)} \varepsilon(v)
$$

where $\varepsilon(v)$ represents eccentricity of vertex $v$.

The average eccentricity avec $(G)$ of a graph $G$ is the mean value of eccentricities of all vertices of a graph, that is,

$$
\operatorname{avec}(G)=\frac{1}{n} \sum_{v \in V(G)} \varepsilon(v) .
$$

The average eccentricity and standard deviation for all Sierpiński graphs $S_{p}^{n}$ is established by [6]. The extremal properties of the average eccentricity, conjectures and Auto graphicx, about the average eccentricity are obtained by [7]. The bounds on the mean eccentricity of a graph, and also the change in mean eccentricity when a graph is replaced by a subgraph, is established by [8]. For trees with fixed diameter, fixed matching number and fixed number of pendent vertices, the lower and upper bounds of average eccentricity are found by [9].

The "eccentricity based geometric-arithmetic $(G A)$ " index of a graph $G$ is defined as [10],

$$
G A_{4}(G)=\sum_{u v \epsilon E(G)} \frac{2 \sqrt{\varepsilon(u) \cdot \varepsilon(v)}}{\varepsilon(u)+\varepsilon(v)} .
$$

Further results regarding the average eccentricity index and eccentricity-based geometric-arithmetic index can be found in [11]. A new version of the $A B C$ index is introduced by Farahani [12] which is defined as,

$$
A B C_{5}(G)=\sum_{u v \in E(G)} \sqrt{\frac{\varepsilon(v)+\varepsilon(u)-2}{\varepsilon(v) \cdot \varepsilon(u)}} .
$$

Imran et al. computed the eccentricity based $A B C$ index and eccentricity based geometric-arithmetic index for copper oxide in [13]. Gao et al. calculated the result about the eccentric $A B C$ index of linear polycene parallelogram benzenoid in [14].

In 2010, D. Vukičević et al. and in 2012, Ghorbani et al. proposed some new modified versions of Zagreb indices of a molecular graph $G[15,16]$. The first Zagreb eccentricity index is defined as:

$$
M_{1}^{*}(G)=\sum_{u v \in E(G)}[\varepsilon(u)+\varepsilon(v)] .
$$

The second Zagreb eccentricity index is defined as:

$$
M_{1}^{* *}(G)=\sum_{v \in V(G)}[\varepsilon(v)]^{2}
$$


The third Zagreb eccentricity index is defined as:

$$
M_{2}^{*}(G)=\sum_{u v \in E(G)} \varepsilon(u) \varepsilon(v)
$$

So, in this article, we extend the study of chemical graph theory to compute the total eccentricity, average eccentricity, eccentricity-based Zagreb indices, $A B C$ index and geometric arithmetic index of oxide network. Furthermore, we give the exact result of these indices which are helpful in studying the underlying topological properties of oxide networks.

\section{Applications of Topological Indices and Motivation}

The $A B C$ index provides a very good correlation for the stability of linear alkanes as well as the branched alkanes and for computing the strain energy of cyclo alkanes [17-20]. To correlate with certain physico-chemical properties, the $G A$ index has much better predictive power than the predictive power of the Randic connectivity index [21-23]. The first and second Zagreb index were found to occur for computation of the total $\pi$-electron energy of the molecules within specific approximate expressions [24].

Since degree based topological indices are useful to analyzed the chemical properties of different molecular structures. So motivated by this idea, we focus on eccentricity based topological indices. As eccentricity based topological indices are used as an important tool to the prediction of physico-chemical, pharmacological and toxicological properties of a compound directly from its molecular structure. This analysis is known as the study of the quantitative structure-activity relationship (QSAR) [25].

\section{Methods}

To compute our results, we use the method of combinatorial computing, vertex partition method, edge partition method, graph theoretical tools, analytic techniques, degree counting method and sum of degrees of neighbours method [26,27]. Moreover, we use Matlab (MathWorks, Natick, MA, USA) for mathematical calculations and verifications (see https:/ / en.wikipedia.org/wiki/MATLAB). We also use the maple software (Maplesoft, McKinney, TX, USA) for plotting these mathematical results (see https://en.wikipedia.org/wiki/Maple_(software)).

\section{Oxide Network}

Oxide networks play a vital role in the study of silicate networks. If we delete silicon vertices from a silicate network, we get an oxide network $O X_{n}$ (see Figure 1). An n-dimensional oxide network is denoted as $O X_{n}$. The number of vertices in Oxide network are $9 n^{2}+3 n$ and number of edges are $18 n^{2}$. An Oxide network $O X_{n}$ with $n=5$ is depicted in Figure 1.

\subsection{Construction of Oxide Network $O X_{n}$ Formulas}

- $\quad$ To prove our main results, we make a partition of vertices of the oxide network $O X_{n}$ for ( $n$-levels) based on eccentricity of each vertex in two sets. The set $V_{1}$ contains those vertices which have the eccentricity $\varepsilon(u)=2 k+1$, and the number of vertices in set $V_{1}$ are $6(2 m-1), 1 \leq m \leq n$. The set $V_{2}$ contain those vertices which have the eccentricity $\varepsilon(u)=2 k+2$, and the number of vertices in set $V_{2}$ are $6 m, 1 \leq m \leq n$. Also, the variable $k$ represents the distance between two vertices, which helps us to make this vertex partition. Also, $k$ represents the range of the total number of vertices with that eccentricity. More preciously, Table 1 represents the vertex partition of Oxide network for (n-levels) based on eccentricity of each vertex. 
- Now we make a partition of edges of an oxide network for ( $n$-levels) based on eccentricity of end vertices in three sets. The set $E_{1}$ contain those edges which have the eccentricities $(\varepsilon(u), \varepsilon(v))=(2 k+1,2 k+1), n \leq k \leq 2 n-1$ and the number of edges in set $E_{1}$ are $6(2 m-1), 1 \leq m \leq n$. The set $E_{2}$ contain those edges which have the eccentricities $(\varepsilon(u), \varepsilon(v))=$ $(2 k+1,2 k+2), n \leq k \leq 2 n-1$, and the number of edges in set $E_{2}$ are $12 m, 1 \leq m \leq n$. The set $E_{3}$ contain those edges which have the eccentricities $(\varepsilon(u), \varepsilon(v))=(2 k+2,2 k+3), n \leq k \leq 2 n-1$, and the number of edges in set $E_{3}$ are $12 m, 1 \leq m \leq n$. Also $k$ represent the range of total number of pairs with that eccentricity. More preciously Table 2 represents the edge partition of oxide network for ( $n$-levels) based on eccentricity of end vertices.

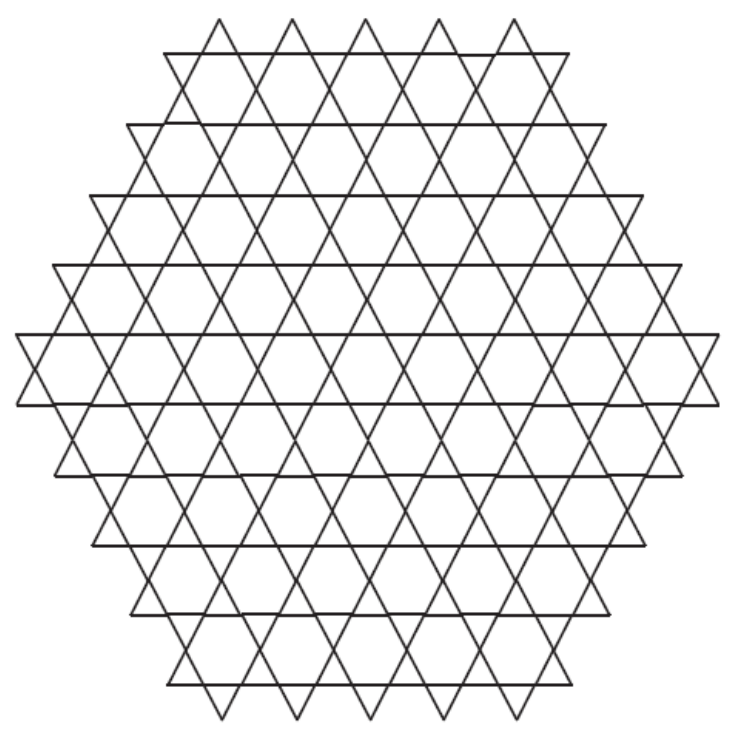

Figure 1. An oxide network $O X_{n}$ with $n=5$.

Table 1. Vertex partition of oxide network for ( $n$-levels) based on eccentricity of each vertex.

\begin{tabular}{ccccc}
\hline$\varepsilon(u)$ & Number of Vertices & Range of $\boldsymbol{k}$ & Range of $\boldsymbol{m}$ and $\boldsymbol{n}$ & Sets \\
\hline $2 k+1$ & $6(2 m-1)$ & $n \leq k \leq 2 n-1$ & $1 \leq m \leq n, n \geq 1$ & $V_{1}$ \\
$2 k+2$ & $6 m$ & $n \leq k \leq 2 n-1$ & $1 \leq m \leq n, n \geq 1$ & $V_{2}$ \\
\hline
\end{tabular}

Table 2. Edge partition of oxide network for ( $n$-levels) based on eccentricity of end vertices.

\begin{tabular}{ccccc}
\hline$(\varepsilon(\boldsymbol{u}), \varepsilon(\boldsymbol{v}))$ & Number of Edges & Range of $\boldsymbol{k}$ & Range of $\boldsymbol{m}$ and $\boldsymbol{n}$ & Sets \\
\hline$(2 k+1,2 k+1)$ & $6(2 m-1)$ & $n \leq k \leq 2 n-1$ & $1 \leq m \leq n, n \geq 1$ & $E_{1}$ \\
$(2 k+1,2 k+2)$ & $12 m$ & $n \leq k \leq 2 n-1$ & $1 \leq m \leq n, n \geq 1$ & $E_{2}$ \\
$(2 k+2,2 k+3)$ & $12 m$ & $n \leq k \leq 2 n-2$ & $1 \leq m \leq n-1, n>1$ & $E_{3}$ \\
\hline
\end{tabular}

\subsection{Main Results for Oxide Network}

In this section, we computed the close formulae of certain topological indices for this network. Here we find the analytically closed results of total eccentricity index, average eccentricity index, eccentricity based Zagreb indices, eccentricity based geometric arithmetic and atom-bond connectivity indices for oxide networks. 
Theorem 1. Let $O X_{n}$, for all $n \in N$, be the oxide network, then the total eccentricity index $\zeta$ of $O X_{n}$ is

$$
\zeta\left(O X_{n}\right)=6 \sum_{m=1}^{n} \sum_{k=n}^{2 n-1}\{6 m k+4 m-2 k-1\} .
$$

Proof. Let $O X_{n}$, where $n \in N$, be the oxide network containing $9 n^{2}+3 n$ vertices and $18 n^{2}$ edges.

Using the vertex partitioned from Table 1 and Equation (2), we have computed the total eccentricity index as:

$$
\begin{aligned}
\zeta(G) & =\sum_{v \in V(G)} \varepsilon(v) \\
\zeta\left(O X_{n}\right) & =\sum_{v \in V_{1}(G)} \varepsilon(v)+\sum_{v \in V_{2}(G)} \varepsilon(v) \\
& =\sum_{m=1}^{n} \sum_{k=n}^{2 n-1} 6(2 m-1) \cdot(2 k+1)+\sum_{m=1}^{n} \sum_{k=n}^{2 n-1} 6 m \cdot(2 k+2) \\
& =6 \sum_{m=1}^{n} \sum_{k=n}^{2 n-1}\{(2 m-1) \cdot(2 k+1)+m \cdot(2 k+2)\}
\end{aligned}
$$

After an easy simplification, we get

$$
\zeta\left(O X_{n}\right)=6 \sum_{m=1}^{n} \sum_{k=n}^{2 n-1}\{6 m k+4 m-2 k-1\}
$$

Theorem 2. Let $O X_{n}$, for all $n \in N$, be the oxide network, then the average eccentricity index avec of $O X_{n}$ is

$$
\operatorname{avec}\left(O X_{n}\right)=\frac{2}{3 n^{2}+n} \sum_{m=1}^{n} \sum_{k=n}^{2 n-1}\{6 m k+4 m-2 k-1\}
$$

Proof. Let $O X_{n}$, where $n \in N$, be the oxide network containing $9 n^{2}+3 n$ vertices and $18 n^{2}$ edges.

Using the vertex partitioned from Table 1 and Equation (3), we have computed the average eccentricity index of oxide network $\operatorname{avec}\left(O X_{n}\right)$ as:

$$
\begin{aligned}
\operatorname{avec}(G) & =\frac{1}{n} \sum_{v \in V(G)} \varepsilon(v) \\
\operatorname{avec}\left(O X_{n}\right) & =\frac{1}{n} \sum_{v \in V_{1}(G)} \varepsilon(v)+\frac{1}{n} \sum_{v \in V_{2}(G)} \varepsilon(v) \\
\operatorname{avec}\left(O X_{n}\right) & =\frac{1}{9 n^{2}+3 n}\left\{\sum_{m=1}^{n} \sum_{k=n}^{2 n-1} 6(2 m-1) \cdot(2 k+1)+\sum_{m=1}^{n} \sum_{k=n}^{2 n-1} 6 m \cdot(2 k+2)\right\}
\end{aligned}
$$

After an easy simplification, we get

$$
\operatorname{avec}\left(O X_{n}\right)=\frac{2}{3 n^{2}+n} \sum_{m=1}^{n} \sum_{k=n}^{2 n-1}\{6 m k+4 m-2 k-1\}
$$


Theorem 3. Let $O X_{n}$ for all $n \in N$, be the oxide network, then the first Zagreb eccentricity index $M_{1}^{*}\left(O X_{n}\right)$ is

$$
M_{1}^{*}\left(O X_{n}\right)=12 \sum_{m=1}^{n} \sum_{k=n}^{2 n-1}\{8 m k+5 m-2 k-1\}+12 \sum_{m=1}^{n-1} \sum_{k=n}^{2 n-2} m(4 k+5)
$$

Proof. Let $O X_{n}$, where $n \in N$, be the oxide network containing $9 n^{2}+3 n$ vertices and $18 n^{2}$ edges.

Using the vertex partitioned from Table 2 and Equation (6), we have computed first Zagreb eccentricity index $M_{1}^{*}\left(O X_{n}\right)$ as:

$$
\begin{aligned}
M_{1}^{*}(G) & =\sum_{u v \in E(G)}[\varepsilon(u)+\varepsilon(v)] \\
M_{1}^{*}\left(O X_{n}\right) & =\sum_{u v \in E_{1}(G)}[\varepsilon(u)+\varepsilon(v)]+\sum_{u v \in E_{2}(G)}[\varepsilon(u)+\varepsilon(v)]+\sum_{u v \in E_{3}(G)}[\varepsilon(u)+\varepsilon(v)] \\
& =\sum_{m=1}^{n} \sum_{k=n}^{2 n-1} 6(2 m-1)(2 k+1+2 k+1)+\sum_{m=1}^{n} \sum_{k=n}^{2 n-2} 12 m(2 k+1+2 k+2) \\
& +\sum_{m=1}^{n-1} \sum_{k=n}^{2 n-2} 12 m(2 k+2+2 k+3) \\
& =6 \sum_{m=1}^{n} \sum_{k=n}^{2 n-1}\{(2 m-1)(4 k+2)+2 m(4 k+3)\}+12 \sum_{m=1}^{n-1} \sum_{k=n}^{2 n-2} m(4 k+5) .
\end{aligned}
$$

After some simplification, we obtain

$$
M_{1}^{*}\left(O X_{n}\right)=12 \sum_{m=1}^{n} \sum_{k=n}^{2 n-1}\{8 m k+5 m-2 k-1\}+12 \sum_{m=1}^{n-1} \sum_{k=n}^{2 n-2} m(4 k+5)
$$

Theorem 4. Let $O X_{n}$ for all $n \in N$, be the oxide network, then the second Zagreb eccentricity index $M_{1}^{* *}\left(O X_{n}\right)$ is

$$
M_{1}^{* *}\left(O X_{n}\right)=6 \sum_{m=1}^{n} \sum_{k=n}^{2 n-1}\left\{2 m\left(6 k^{2}+8 k+3\right)-(2 k+1)^{2}\right\}
$$

Proof. Let $O X_{n}$, where $n \in N$, be the oxide network containing $9 n^{2}+3 n$ vertices and $18 n^{2}$ edges.

The general formula of second Zagreb eccentricity index is

$$
\begin{gathered}
M_{1}^{* *}(G)=\sum_{v \in V(G)}[\varepsilon(v)]^{2} . \\
M_{1}^{* *}(G)=\sum_{v \in V_{1}(G)}[\varepsilon(v)]^{2}+\sum_{v \in V_{2}(G)}[\varepsilon(v)]^{2} .
\end{gathered}
$$

By using the values from Table 1 , we have

$$
\begin{gathered}
M_{1}^{* *}\left(O X_{n}\right)=\sum_{m=1}^{n} \sum_{k=n}^{2 n-1} 6(2 m-1) \cdot(2 k+1)^{2}+\sum_{m=1}^{n} \sum_{k=n}^{2 n-1} 6 m \cdot(2 k+2)^{2} . \\
M_{1}^{* *}\left(O X_{n}\right)=6 \sum_{m=1}^{n} \sum_{k=n}^{2 n-1}\left\{(2 m-1) \cdot(2 k+1)^{2}+4 m \cdot(k+1)^{2}\right\} .
\end{gathered}
$$


After some simplification, we obtain

$$
M_{1}^{* *}\left(O X_{n}\right)=6 \sum_{m=1}^{n} \sum_{k=n}^{2 n-1}\left\{2 m\left(6 k^{2}+8 k+3\right)-(2 k+1)^{2}\right\}
$$

Theorem 5. Let $O X_{n}$ for all $n \in N$, be the oxide network, then the third Zagreb eccentricity index $M_{2}^{*}\left(O X_{n}\right)$ is

$$
M_{2}^{*}\left(O X_{n}\right)=12 \sum_{m=1}^{n} \sum_{k=n}^{2 n-1}\left\{2 m\left(8 k^{2}+8 k+3\right)-\left(4 k^{2}+2 k+1\right)\right\}+24 \sum_{m=1}^{n-1} \sum_{k=n}^{2 n-2} m(2 k+3)(k+1) .
$$

Proof. Let $O X_{n}$, where $n \in N$, be the oxide network containing $9 n^{2}+3 n$ vertices and $18 n^{2}$ edges.

The general formula of third Zagreb eccentricity index is

$$
\begin{gathered}
M_{2}^{*}(G)=\sum_{u v \in E(G)}[\varepsilon(u) \cdot \varepsilon(v)] . \\
M_{2}^{*}(G)=\sum_{u v \in E_{1}(G)}[\varepsilon(u) \cdot \varepsilon(v)]+\sum_{u v \in E_{2}(G)}[\varepsilon(u) \cdot \varepsilon(v)]+\sum_{u v \in E_{3}(G)}[\varepsilon(u) \cdot \varepsilon(v)]
\end{gathered}
$$

By using the values from Table 2, we have

$$
\begin{gathered}
M_{2}^{*}\left(O X_{n}\right)=\sum_{m=1}^{n} \sum_{k=n}^{2 n-1} 6(2 m-1)(2 k+1) \cdot(2 k+1)+\sum_{m=1}^{n} \sum_{k=n}^{2 n-1} 12 m(2 k+1) \cdot(2 k+2) \\
\quad+\sum_{m=1}^{n-1} \sum_{k=n}^{2 n-2} 12 m(2 k+2) \cdot(2 k+3) \\
M_{2}^{*}\left(O X_{n}\right)=6 \sum_{m=1}^{n} \sum_{k=n}^{2 n-1}\left\{(2 m-1)(2 k+1)^{2}+2 m\left(4 k^{2}+4 k+2 k+2\right)\right\}+24 \sum_{m=1}^{n-1} \sum_{k=n}^{2 n-2} m(k+1)(2 k+3)
\end{gathered}
$$

After some simplification, we obtain

$$
M_{2}^{*}\left(O X_{n}\right)=12 \sum_{m=1}^{n} \sum_{k=n}^{2 n-1}\left\{2 m\left(8 k^{2}+8 k+3\right)-\left(4 k^{2}+2 k+1\right)\right\}+24 \sum_{m=1}^{n-1} \sum_{k=n}^{2 n-2} m(2 k+3)(k+1) .
$$

Theorem 6. Let $O X_{n}$ for all $n \in N$, be the oxide network, then the geometric-arithmetic index $G_{4}\left(O X_{n}\right)$ is

$$
\begin{aligned}
G A_{4}\left(O X_{n}\right) & =12 \sum_{m=1}^{n} \sum_{k=n}^{2 n-1}\left\{\frac{2 m-1}{2}+2 m \frac{\sqrt{(2 k+1)(2 k+2)}}{(4 k+3)}\right\} \\
& +24 \sum_{m=1}^{n-1} \sum_{k=n}^{2 n-2} m \sqrt{\frac{(2 k+2)(2 k+3)}{4 k+5}}
\end{aligned}
$$

Proof. Let $O X_{n}$, where $n \in N$, be the oxide network containing $9 n^{2}+3 n$ vertices and $18 n^{2}$ edges. The general formula of eccentricity based geometric arithmetic index is

$$
G A_{4}(G)=\sum_{u v \in E(G)} \frac{2 \sqrt{\varepsilon(u) \cdot \varepsilon(v)}}{\varepsilon(u)+\varepsilon(v)}
$$




$$
G A_{4}(G)=\sum_{u v \in E_{1}(G)} \frac{2 \sqrt{\varepsilon(u) \cdot \varepsilon(v)}}{\varepsilon(u)+\varepsilon(v)}+\sum_{u v \epsilon E_{2}(G)} \frac{2 \sqrt{\varepsilon(u) \cdot \varepsilon(v)}}{\varepsilon(u)+\varepsilon(v)}+\sum_{u v \in E_{3}(G)} \frac{2 \sqrt{\varepsilon(u) \cdot \varepsilon(v)}}{\varepsilon(u)+\varepsilon(v)}
$$

Using the edge partitioned from Table 2, we have the following computations

$$
\begin{gathered}
G A_{4}(O X n)=\sum_{m=1}^{n} \sum_{k=n}^{2 n-1} 6(2 m-1) \cdot 2 \frac{\sqrt{(2 k+1) \cdot(2 k+1)}}{2 k+1+2 k+1}+\sum_{m=1}^{n} \sum_{k=n}^{2 n-1} 12 m \cdot 2 \frac{\sqrt{(2 k+1) \cdot(2 k+2)}}{2 k+1+2 k+2} \\
+\sum_{m=1}^{n-1} \sum_{k=n}^{2 n-2} 12 m \cdot 2 \frac{\sqrt{(2 k+2) \cdot(2 k+3)}}{2 k+2+2 k+3} . \\
G A_{4}(O X n)=12 \sum_{m=1}^{n} \sum_{k=n}^{2 n-1}\left\{(2 m-1) \frac{\sqrt{(2 k+1)^{2}}}{4 k+2}+2 \frac{\sqrt{(2 k+1) \cdot(2 k+2)}}{4 k+3}\right\} \\
+24 \sum_{m=1}^{n-1} \sum_{k=n}^{2 n-2} m \frac{\sqrt{(2 k+2) \cdot(2 k+3)}}{4 k+5} .
\end{gathered}
$$

After some simplification, we obtain

$$
\begin{aligned}
G A_{4}\left(O X_{n}\right) & =12 \sum_{m=1}^{n} \sum_{k=n}^{2 n-1}\left\{\frac{2 m-1}{2}+2 m \frac{\sqrt{(2 k+1)(2 k+2)}}{(4 k+3)}\right\} \\
& +24 \sum_{m=1}^{n-1} \sum_{k=n}^{2 n-2} m \sqrt{\frac{(2 k+2)(2 k+3)}{4 k+5}}
\end{aligned}
$$

Theorem 7. Let $O X_{n}$ for all $n \in N$, be the oxide network, then the atom-bond connectivity index $A B C_{5}\left(O X_{n}\right)$ is

$$
\begin{aligned}
A B C_{5}\left(O X_{n}\right) & =12 \sum_{m=1}^{n} \sum_{k=n}^{2 n-1}\left\{\frac{(2 m-1) \sqrt{k}}{2 k+1}+m \sqrt{\frac{4 k+1}{(2 k+1)(2 k+2)}}\right\} \\
& +12 \sum_{m=1}^{n-1} \sum_{k=n}^{2 n-2} m \sqrt{\frac{4 k+3}{(2 k+2)(2 k+3)}}
\end{aligned}
$$

Proof. Let $O X_{n}$, where $n \in N$, be the oxide network containing $9 n^{2}+3 n$ vertices and $18 n^{2}$ edges.

The general formula of eccentricity based atom-bond connectivity index is

$$
\begin{gathered}
A B C_{5}(G)=\sum_{u v \in E(G)} \sqrt{\frac{\varepsilon(u)+\varepsilon(v)-2}{\varepsilon(u) \cdot \varepsilon(v)}} . \\
A B C_{5}(G)=\sum_{u v \in E_{1}(G)} \sqrt{\frac{\varepsilon(u)+\varepsilon(v)-2}{\varepsilon(u) \cdot \varepsilon(v)}}+\sum_{u v \in E_{2}(G)} \sqrt{\frac{\varepsilon(u)+\varepsilon(v)-2}{\varepsilon(u) \cdot \varepsilon(v)}}+\sum_{u v \in E_{3}(G)} \sqrt{\frac{\varepsilon(u)+\varepsilon(v)-2}{\varepsilon(u) \cdot \varepsilon(v)}} .
\end{gathered}
$$


Using the edge partitioned from Table 2, we have the following computations

$$
\begin{gathered}
A B C_{5}\left(O X_{n}\right)=\sum_{m=1}^{n} \sum_{k=n}^{2 n-1} 6(2 m-1) \sqrt{\frac{2 k+1+2 k+1-2}{(2 k+1) \cdot(2 k+1)}}+\sum_{m=1}^{n} \sum_{k=n}^{2 n-1} 12 m \sqrt{\frac{2 k+1+2 k+2-2}{(2 k+1) \cdot(2 k+2)}} \\
+\sum_{m=1}^{n-1} \sum_{k=n}^{2 n-2} 12 m \sqrt{\frac{2 k+2+2 k+3-2}{(2 k+2) \cdot(2 k+3)}} \\
A B C_{5}\left(O X_{n}\right)=6 \sum_{m=1}^{n} \sum_{k=n}^{2 n-1}\left\{(2 m-1) \sqrt{\frac{4 k}{(2 k+1)^{2}}}+2 m \sqrt{\frac{4 k+1}{(2 k+1) \cdot(2 k+2)}}\right\} \\
+12 \sum_{m=1}^{n-1} \sum_{k=n}^{2 n-2} m \sqrt{\frac{4 k+3}{(2 k+2) \cdot(2 k+3)}} .
\end{gathered}
$$

After some simplification, we obtain

$$
\begin{aligned}
A B C_{5}\left(O X_{n}\right) & =12 \sum_{m=1}^{n} \sum_{k=n}^{2 n-1}\left\{\frac{(2 m-1) \sqrt{k}}{2 k+1}+m \sqrt{\frac{4 k+1}{(2 k+1)(2 k+2)}}\right\} \\
& +12 \sum_{m=1}^{n-1} \sum_{k=n}^{2 n-2} m \sqrt{\frac{4 k+3}{(2 k+2)(2 k+3)}} .
\end{aligned}
$$

\section{Comparisons and Discussion}

For the comparison of these indices numerically for $O X_{n}$, we computed all indices for different values of $m, k$. Now, from Table 3, we can easily see that all indices are in increasing order as the values of $m, k$ are increasing. The graphical representations of the all indices for $O X_{n}$ are depicted in Figures 2-8 for certain values of $m, k$.

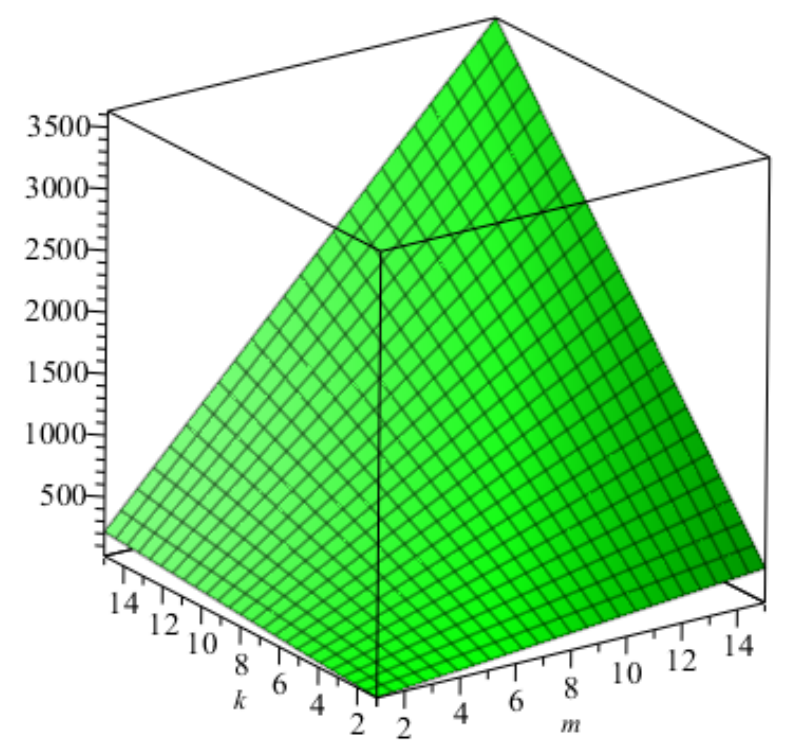

Figure 2. The graphically representation of total eccentricity index $\zeta$ of $O X_{n}$. 
Table 3. Numerical computation of all indices for $O X_{n}$.

\begin{tabular}{cccccccc}
\hline$[m, k]$ & $\zeta(G)$ & avec $(G)$ & $M_{1}^{*}(G)$ & $\boldsymbol{M}_{1}^{* *}(G)$ & $\boldsymbol{M}_{2}^{*}(G)$ & $G A_{4}(G)$ & $A B C_{5}(G)$ \\
\hline$[1,1]$ & 42 & 1.9 & 1416 & 2568 & 2014 & 112.5 & 315.4 \\
{$[2,2]$} & 162 & 3.5 & 4188 & 5478 & 4352 & 279.9 & 645.3 \\
{$[3,3]$} & 354 & 5.6 & 8304 & 10,523 & 9300 & 446.7 & 987.4 \\
{$[4,4]$} & 618 & 8.4 & 13,764 & 14,587 & 11,248 & 613.6 & 1125.6 \\
{$[5,5]$} & 956 & 10.5 & 16,898 & 16,325 & 13,654 & 842.3 & 1356.4 \\
{$[6,6]$} & 1242 & 14.5 & 19,652 & 19,876 & 16,324 & 1023.3 & 1586.7 \\
\hline
\end{tabular}

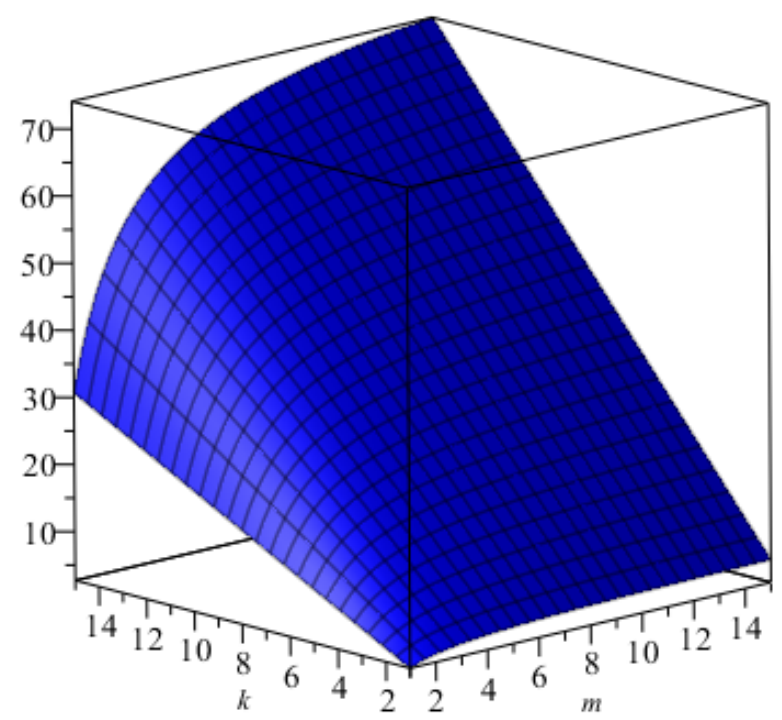

Figure 3. The graphically representation of the average eccentricity index avec of $O X_{n}$.

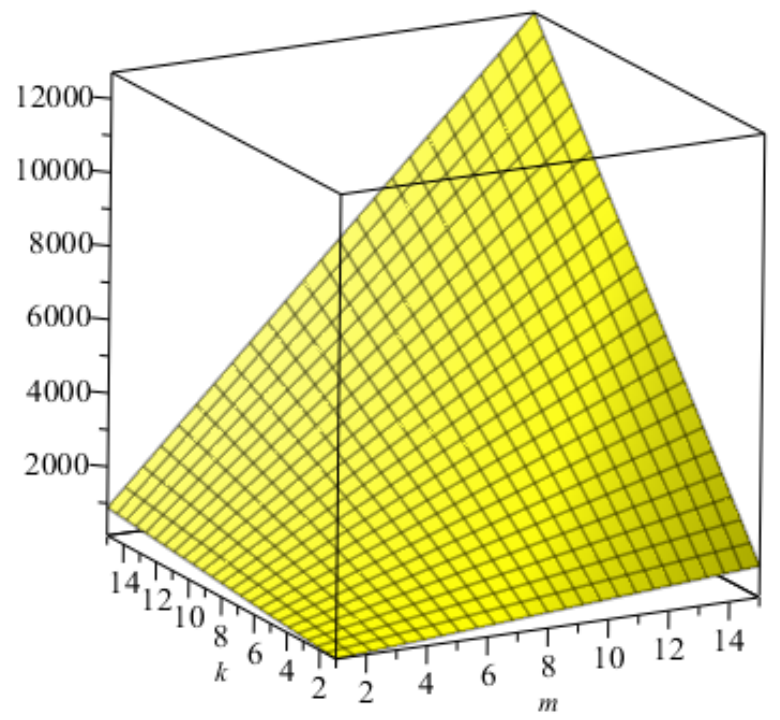

Figure 4. The graphically representation of the first Zagreb eccentricity index $M_{1}^{*}\left(O X_{n}\right)$. 


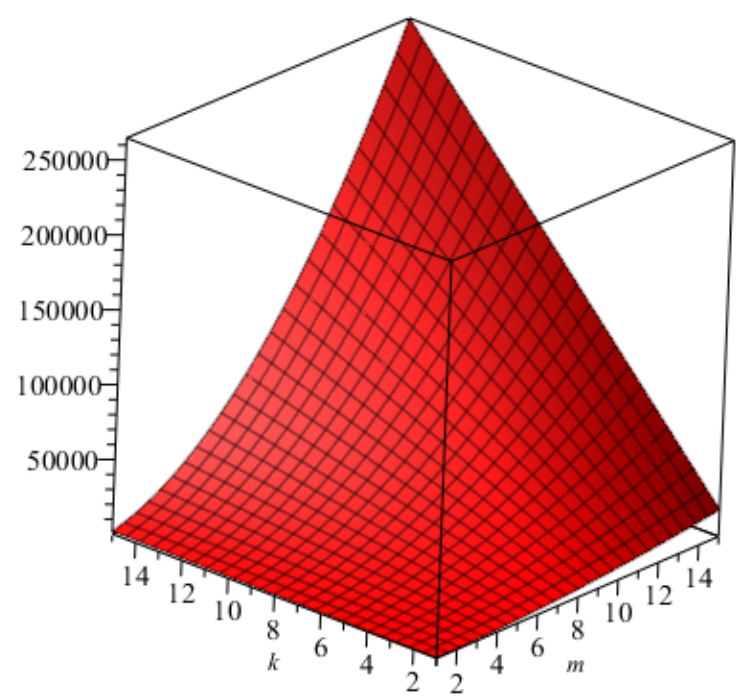

Figure 5. The graphically representation of the second Zagreb eccentricity index $M_{1}^{* *}\left(O X_{n}\right)$.

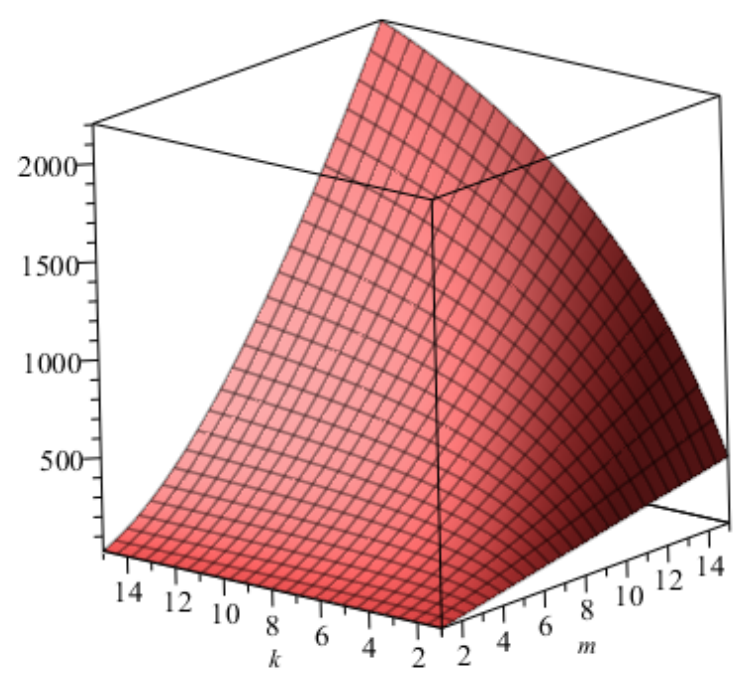

Figure 6. The graphically representation of the third Zagreb eccentricity index $M_{1}^{* *}\left(O X_{n}\right)$.

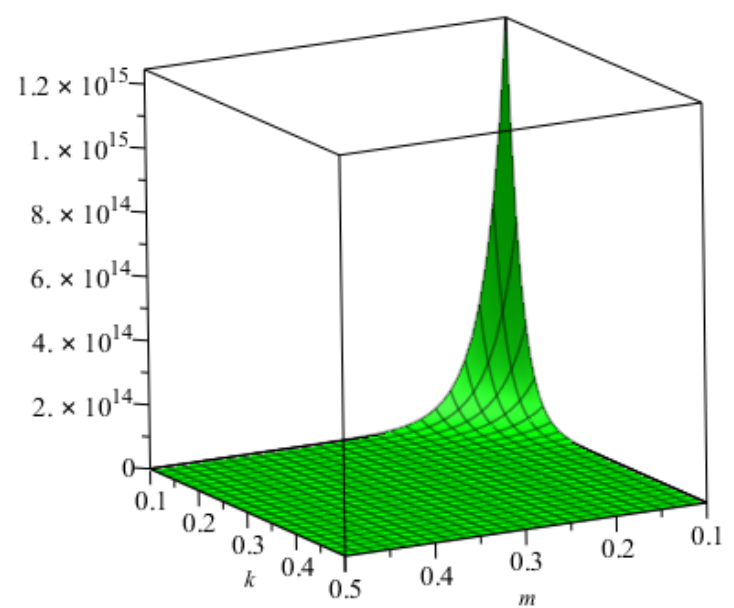

Figure 7. The graphically representation of the geometric-arithmetic index $G A_{4}\left(O X_{n}\right)$. 


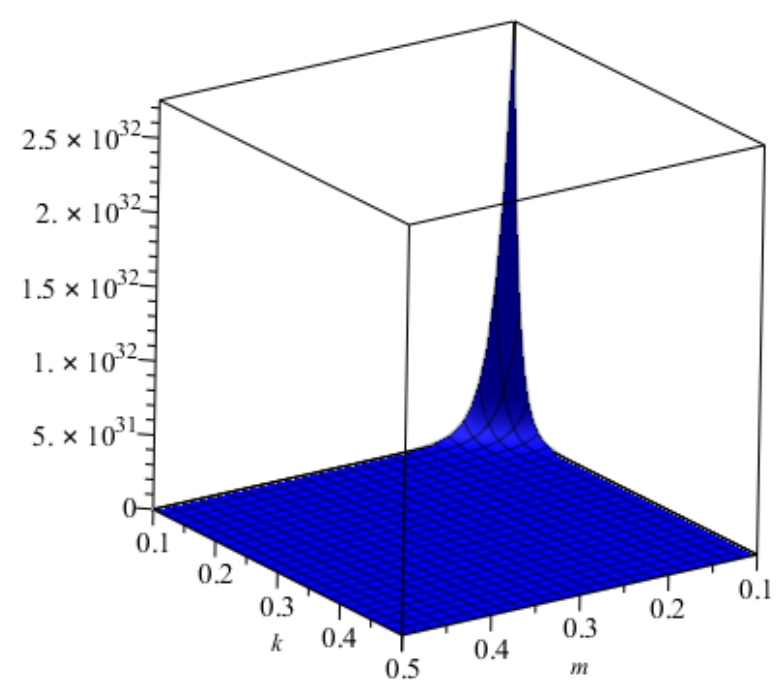

Figure 8. The graphically representation of the atom-bond connectivity index $A B C_{5}\left(O X_{n}\right)$.

\section{Conclusions}

In this paper, we computed the total eccentricity index $\zeta\left(O X_{n}\right)$, average eccentricity index avec $\left(O X_{n}\right)$, eccentricity-based Zagreb indices $M_{1}^{*}\left(O X_{n}\right), M_{1}^{* *}\left(O X_{n}\right)$ and $M_{2}^{*}\left(O X_{n}\right)$, atom-bond connectivity index $A B C_{5}\left(O X_{n}\right)$ and geometric arithmetic index $G A_{4}\left(O X_{n}\right)$ of the oxide network $O X_{n}$. So these indices are useful to analyzed the physico-chemical, pharmacological and toxicological properties of the oxide network $O X_{n}$.

Author Contributions: M.I. contribute for supervision, project administration, funding and analyzed the data curation. M.K.S. and A.Q.B. contribute for designing the experiments, validation, conceptualization and formal analysing. A.A.E.A. and D.A. contribute for performed experiments, resources, software and some computations. S.H.R. contribute for Investigation, Methodology and wrote the initial draft of the paper which were investigated and approved by M.I. and wrote the final draft. All authors read and approved the final version of the paper.

Acknowledgments: The authors are grateful to the anonymous referees for their valuable comments and suggestions that improved this paper. This research is supported by the Start-Up Research Grant 2016 of United Arab Emirates University (UAEU), Al Ain, United Arab Emirates via Grant No. G00002233, UPAR Grant of UAEU via Grant No. G00002590 and by the Summer Undergraduate Research Experience (SURE) plus 2017 research Grant via Grant No. G00002412.

Conflicts of Interest: The authors declare no conflict of interest.

\section{References}

1. Hayat, S.; Malik, M.A.; Imran, M. Computing topological indices of honeycomb derived networks. Romanian J. Inf. Sci. Technol. 2015, 18, 144-165.

2. Imran, M.; Ali, M.A.; Ahmad, S.; Siddiqui, M.K.; Baig, A.Q. Topological sharacterization of the symmetrical structure of bismuth tri-iodide. Symmetry 2018, 10, 201. [CrossRef]

3. Bie, R.J.; Siddiqui, M.K.; Razavi, R.; Taherkhani, M.; Najaf, M. Possibility of $\mathrm{C}_{38}$ and $\mathrm{Si}_{19} \mathrm{Ge}_{19}$ nanocages in anode of metal ion batteries: Computational examination. Acta Chim. Slov. 2018, 65, 303-311. [CrossRef] [PubMed]

4. Gao, W.; Siddiqui, M.K. Molecular descriptors of nanotube, oxide, silicate, and triangulene networks. J. Chem. 2017, 2017, 6540754. [CrossRef]

5. Farooq, R.; Malik, M.A. On some eccentricity based topological indices of nanostar dendrimers. 2018, in press.

6. Hinz, A.M.; Parisse, D. The Average Eccentricity of Sierpinski Graphs. Graphs Comb. 2012, 5, 671-686. [CrossRef]

7. Ilic, A. On the extremal properties of the average eccentricity. Comput. Math. Appl. 2012, 64, 2877-2885. [CrossRef] 
8. Dankelmann, P.; Goddard, W.; Swart, C.S. The average eccentricity of a graph and its subgraphs. Util. Math. 2004, 65, 41-51.

9. Tang, Y.; Zhou, B. On average eccentricity. MATCH Commun. Math. Comput. Chem. 2012, 67, 405-423.

10. Ghorbani, M.; Khaki, A. A note on the fourth version of geometric-arithmetic index. Optoelectron. Adv. Mater. Rapid Commun. 2010, 4, 2212-2215.

11. Zhang, X.; Baig, A.Q.; Azhar, M.R.; Farahani, M.R.; Imran, M. The Average eccentricity and Eccentricity based Geometric-arithmetic index of tetra sheets. Int. J. Pure Appl. Math. 2017, 117, 467-479.

12. Farahani, M.R. Eccentricity version of atom bond connectivity index of benzenoid family $A B C_{5}(H k)$. World Appl. Sci. J. Chem. 2013, 21, 1260-1265.

13. Imran, M.; Baig, A.Q.; Azhar, M.R.; Farahani, M.R.; Zhang, X. Eccentricity based geometric-arithmetic and atom-bond connectivity indices of copper oxide CuO. Int. J. Pure Appl. Math. 2017, 117, 481-502.

14. Gao, W.; Farahani, M.R.; Jamil, M.K. The eccentricity version of atom-bond connectivity index of linear polycene parallelogram benzoid $A B C_{5}(P(n, n))$. Acta Chim. Slov. 2016, 63, 376-379. [CrossRef] [PubMed]

15. Ghorbani, M.; Hosseinzadeh, M.A. A new version of Zagreb indices. Filomat 2012, 6, 93-100. [CrossRef]

16. Vukičević, D.; Graovac, A. Note on the comparison of the first and second normalized Zagreb eccentricity indices. Acta Chim. Slov. 2010, 57, 524-528.

17. Estrada, E.; Torres, L.; Rodríguez, L.; Gutman, I. An atom-bond connectivity inde. Modelling the enthalpy of formation of alkanes. Indian J. Chem. 1998, 37, 849-855.

18. Gao, W.; Siddiqui, M.K.; Naeem, M.; Rehman, N.A. Topological Characterization of Carbon Graphite and Crystal Cubic Carbon Structures. Molecules 2017, 22, 1496. [CrossRef] [PubMed]

19. Siddiqui, M.K.; Naeem, M.; Rahman, N.A.; Imran, M. Computing topological indicesof certain networks. J. Optoelectron. Adv. Mater. 2016, 18, 884-892.

20. Shao, Z.; Wu, P.; Zhang, X.; Dimitrov, D.; Liu, J. On the maximum ABC index of graphs with prescribed size and without pendent vertices. IEEE Access 2018, 6, 27604-27616. [CrossRef]

21. Siddiqui, M.K.; Imran, M.; Ahmad, A. On Zagreb indices, Zagreb polynomials of some nanostar dendrimers. Appl. Math. Comput. 2016, 280, 132-139. [CrossRef]

22. Shao, Z.; Wu, P.; Gao, Y.; Gutman, I.; Zhang, X. On the maximum ABC index of graphs without pendent vertices. Appl. Math. Comput. 2017, 315, 298-312. [CrossRef]

23. Baig, A.Q.; Imran, M.; Ali, H. On topological indices of poly oxide, poly silicate, DOX, and DSL networks. Can. J. Chem. 2015, 93, 730-739. [CrossRef]

24. Shao, Z.; Siddiqui, M.K.; Muhammad, M.H. Computing zagreb indices and zagreb polynomials for symmetrical nanotubes. Symmetry 2018, 10, 244. [CrossRef]

25. Gupta, S.; Singh, M.; Madan, A.K. Application of Graph Theory: Relationship of Eccentric Connectivity Index and Wiener's Index with Anti-inflammatory Activity. J. Math. Anal. Appl. 2002, 266, 259-268. [CrossRef]

26. Chartrand, G.; Zhang, P. Introduction to Graph Theory; McGraw-Hill Higher Education: New York, NY, USA, 2007.

27. Harris, J.M.; Hirst, J.L.; Mossinghoff, M.J. Combinatorics and Graph Theory; Springer Science and Business Media: Berlin/Heidelberg, Germany, 2008.

(C) 2018 by the authors. Licensee MDPI, Basel, Switzerland. This article is an open access article distributed under the terms and conditions of the Creative Commons Attribution (CC BY) license (http:// creativecommons.org/licenses/by/4.0/). 